\title{
Dynamic stabilization of nonequilibrium domain configurations in magnetic squares with high amplitude excitations
}

\author{
S. E. Stevenson, ${ }^{1}$ C. Moutafis, ${ }^{1}$ G. Heldt,${ }^{1,2}$ R. V. Chopdekar, ${ }^{1}$ C. Quitmann, ${ }^{1}$ L. J. Heyderman,${ }^{1,2}$ and J. Raabe ${ }^{1}$ \\ ${ }^{1}$ Paul Scherrer Institut, 5232 Villigen PSI, Switzerland \\ ${ }^{2}$ Laboratory for Mesoscopic Systems, Department of Materials, ETH Zurich, Wolfgang-Pauli-Strasse 10, 8093 Zurich, Switzerland
}

(Received 9 November 2012; revised manuscript received 25 January 2013; published 19 February 2013)

\begin{abstract}
We explore the linear and nonlinear dynamic regimes of micrometer-scale soft magnetic squares with an in-plane uniaxial anisotropy, recording the response of the magnetization in the spatial domain under a continuous sinusoidal excitation with time-resolved scanning transmission x-ray microscopy. Increasing the excitation field amplitude leads to the dynamic stabilization of nonequilibrium domain configurations, which appear at threshold amplitudes and return to the equilibrium configuration when the amplitude is reduced. On imaging the magnetization in the transition region between two stable magnetic configurations, we observe small domains originating from the vortex core. Dynamic micromagnetic simulations of the domain configuration provide qualitative agreement with the experimental data and insight into the energy dissipation and spin wave contributions.
\end{abstract}

DOI: 10.1103/PhysRevB.87.054423

PACS number(s): 75.78.-n, 05.45.-a, 75.50.Bb, 75.70.Kw

\section{INTRODUCTION}

Time-resolved studies of magnetic domain wall, vortex and spin dynamics in mesoscopic thin film structures have become of great interest in recent years due to their importance in advancing both fundamental micromagnetics understanding and potential commercial applications. Indeed, there have been several advances toward the use of magnetic vortex cores as memory components, where the bistability of the core polarity allows for binary data storage. ${ }^{1-3}$ Multiple eigenmodes (spin wave modes) and the vortex core gyration mode have been established for different resonant frequencies, which are determined by the dimensions of the magnetic structure. $^{4-8}$

It has been shown that when the amplitude of an applied excitation field is increased, the observed behavior of the magnetization changes from the linear to the nonlinear regime. ${ }^{8-10}$ A number of these studies have focused on the influence of the excitation amplitude for different eigenmodes, and on increasing the excitation amplitude higher-order modes are induced. ${ }^{11,12}$ At the onset of the nonlinear regime there have been some interesting observations at the vortex core with reports of distortions to the vortex core coupled with random switching of the core polarity. ${ }^{13}$ Spin wave instabilities in nonequilibrium modes, which arise in the nonlinear regime, ${ }^{6,14-16}$ can ultimately lead to vortex core reversal.

To control vortex core reversal in a variety of systems it is of paramount interest to understand the relevant nonlinear processes. Therefore, to reveal the details of this nonlinear behavior, we investigate the effects of high amplitude excitations on micrometer-scale permalloy $\left(\mathrm{Ni}_{81} \mathrm{Fe}_{19}\right)$ magnetic squares, specifically in the spatial domain. For this we use synchrotron-based soft $\mathrm{x}$-ray microscopy, which is an ideal tool for the study of magnetization dynamics due to its high spatial and temporal resolution. ${ }^{17-21}$ In particular, we employ scanning transmission x-ray microscopy (STXM), an element-selective pump-probe technique. ${ }^{22-25}$ We carry out time-resolved studies of the magnetization dynamics in the linear and nonlinear regimes of the permalloy squares with an in-plane anisotropy. At equilibrium, these squares present a Landau flux closure state with an elongated domain wall at the vortex core. Micromagnetic simulations indicate that the elongation of this domain wall is due to the presence of a uniaxial anisotropy $K_{x}$.

We apply continuous-wave (CW) excitations, observing the translational oscillations of the domain configurations. At low amplitudes, the dynamics of the equilibrium mode are observed. When the amplitude is increased to threshold values, we observe nonequilibrium modes. We compare our experimental observations to micromagnetic simulations, which show good agreement and aid the understanding of the dynamic processes.

\section{SAMPLE PREPARATION, MEASUREMENTS, AND SIMULATIONS}

\section{A. Fabrication of magnetic squares}

A schematic of a typical sample is shown in Fig. 1(a). Square magnetic structures with side lengths $\ell=4,6$, and $8 \mu \mathrm{m}$ were fabricated using electron beam lithography and lift-off processing on a prepatterned silicon $(\mathrm{Si})$ substrate with a silicon nitride $\left(\mathrm{Si}_{3} \mathrm{~N}_{4}\right)$ window which was etched to form a membrane following the lithography in a potassium hydroxide $(\mathrm{KOH})$ solution. During a first lithographic step, permalloy (thickness, $\tau=40 \mathrm{~nm}$ ), with an aluminum (Al) capping layer ( $\tau=1 \mathrm{~nm}$ ) to prevent the oxidation of the magnetic material, was deposited by DC magnetron sputtering. An electrically conductive copper stripline $(\mathrm{Cu}, \tau=200 \mathrm{~nm}$, width $10 \mu \mathrm{m})$ for the application of the magnetic field excitation is deposited on top of the structures by thermal evaporation during a second lithographic step with adhesion and capping layers of chromium $(\mathrm{Cr}, \tau=5 \mathrm{~nm})$. A further $150 \mathrm{~nm}$ of thermally conductive aluminum nitride (AlN) was coated over the samples to aid heat dissipation from the stripline during excitation. The substrate was subsequently etched from the back in a solution of $20 \% \mathrm{KOH}$ to form the $\mathrm{x}$-ray transparent $\mathrm{Si}_{3} \mathrm{~N}_{4}$ membrane $(\tau=100 \mathrm{~nm})$.

To avoid excess heating during excitation, the resistance of the stripline should be as low as possible. We measured a 
(a)

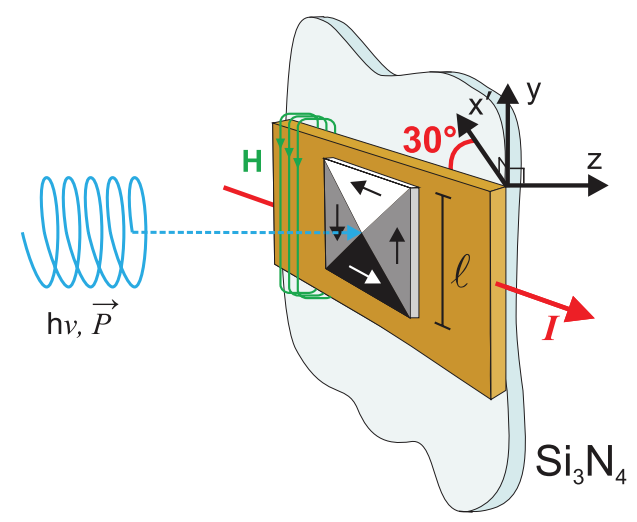

(b)

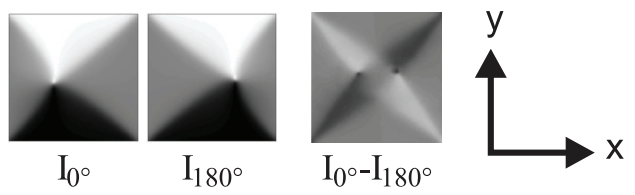

FIG. 1. (Color online) (a) Schematic indicating a section of $\mathrm{Si}_{3} \mathrm{~N}_{4}$ membrane with a square magnetic structure of side length $\ell$ in the Landau state on a conductive stripline. Sinusoidal current $I$ passing through the stripline induces an excitation field $\vec{H}$. The polarization vector $\vec{P}$ is along $z$. The sample is tilted at an angle of $30^{\circ}$ to the $x^{\prime} y$ plane. (b) Schematic of XMCD images $I_{0^{\circ}}$ and $I_{180^{\circ}}$ showing the domain configuration with vortex displacement at $0^{\circ}$ and $180^{\circ}$ of the sinusoidal field excitation. The contrast indicates the intensity of $\vec{M}_{x}$. The phase difference image $\left(I_{0^{\circ}}-I_{180^{\circ}}\right)$ isolates the time-dependent effects.

resistance of $R_{\mathrm{DC}}=13.2 \Omega$ through the connected stripline, which is an acceptable value allowing for our high amplitude excitations. A sinusoidal current passing through the stripline will induce an oscillating magnetic field $\vec{H}=\vec{H}_{0} \cos (\omega t+\phi)$ causing the excitation of the magnetization in our samples.

\section{B. Magnetic x-ray microscopy}

Imaging of the magnetization in our structures was carried out using element specific STXM at the PolLux beamline of the Swiss Light Source. ${ }^{26}$ All images were recorded at the Ni $\mathrm{L}_{3}$ absorption edge $E=854.5 \mathrm{eV}$. Circularly polarized x-rays are obtained from a bending magnet and the monochromatic beam is focused by a Fresnel zone plate (FZP) allowing a spatial resolution down to $15 \mathrm{~nm} .{ }^{27}$ The sample is raster scanned through the FZP focal point and the transmitted intensity is measured by a fast avalanche photodiode with single photon sensitivity. ${ }^{28}$ For sensitivity to the in-plane component of magnetization $\vec{M}_{x}$, the sample is tilted $30^{\circ}$ to the $x^{\prime} y$ plane [Fig. 1(a)]. Magnetic contrast is obtained via the $\mathrm{x}$-ray magnetic circular dichroism (XMCD) effect, ${ }^{29,30}$ where the transmitted $\mathrm{x}$-ray intensity depends on the orientation of magnetization $\vec{M}$ with respect to the polarization $\vec{P}$. A static XMCD image is acquired prior to the application of an excitation field to obtain the initial equilibrium magnetization configuration. A ratio of two images acquired with opposite polarizations $(\mathrm{C}+$ and $\mathrm{C}-$ ) removes the topographic contrast and other artifacts, yielding the desired magnetic information: $I_{\mathrm{XMCD}}=\frac{I_{\mathrm{C}+(\vec{r})-I_{\mathrm{C}-}(\vec{r})}}{I_{\mathrm{C}+}(\vec{r})+I_{\mathrm{C}-}(\vec{r})} \approx \frac{I_{\mathrm{C}+(\vec{r})}}{I_{\mathrm{C}-}(\vec{r})} \approx \vec{M}(\vec{r}) \vec{P}$.
Time-resolved measurements allow the direct imaging of the magnetization dynamics in our samples. The limit of temporal resolution is given by the bunch length of the x-rays, 70 ps (FWHM). A sinusoidal CW excitation $\vec{I}(t)=\vec{I}_{0}$ $\cos (\omega t+\phi)$, synchronous to the $\mathrm{x}$-ray bunch pattern, was applied through the stripline. The frequency, which must be a subharmonic of the $f_{M}=500 \mathrm{MHz}$ master x-ray repetition rate, was chosen to be $f=250 \mathrm{MHz}$, which is close to the resonance frequency of our magnetic squares, thus maximizing the dynamic response. The applied $\mathrm{CW}$ excitation induces an oscillating magnetic field $\vec{H}(t)=\vec{H}_{0} \sqrt{2} \cos (\omega t+\phi)$, where $\vec{H}_{0}$ is the RMS value of the magnetic field and is applied up to $3.2 \mathrm{mT}$ in the plane of the magnetic squares along $y$ [Fig. 1(a)]. To improve heat dissipation during dynamic experiments, the chamber of the microscope was filled with 500-mbar helium.

For a continuous $250-\mathrm{MHz}$ excitation with a 4-ns period, two images are acquired with 2-ns spacing. By shifting the phase of the excitation signal by $90^{\circ}(=1 \mathrm{~ns})$ we acquire four images with 1-ns spacing. These are temporal XMCD images $I_{\mathrm{XMCD}}(\phi, \vec{r})$ showing the magnetic configuration corresponding to phases $0^{\circ}, 90^{\circ}, 180^{\circ}$, and $270^{\circ}$ of the sinusoidal excitation field. Thus, we can follow the deterministic temporal response of the system. Temporal XMCD images are obtained similarly to the static XMCD images, from the ratio of oppositely polarized images acquired at the same excitation phase. The time-dependent effects are captured in phase difference images which display the extent of in-plane dynamics, as schematically shown in Fig. 1(b). These phase difference images $I^{*}(\vec{r})=\frac{I_{0^{\circ}}(\vec{r})}{I_{180^{\circ}}(\vec{r})} \approx\left(I_{0^{\circ}}-I_{180^{\circ}}\right)$ are obtained from the ratio of data recorded simultaneously from two signal channels, $180^{\circ}$ (=2 ns) apart, using only $\mathrm{C}-$ light.

The equilibrium configurations of soft magnetic mesoscopic square and circular thin-film structures are vortex flux-closure states. In squares specifically, the equilibrium magnetic configuration is a Landau state. This consists of four triangular domains with uniform in-plane magnetization, separated by domain walls which meet at the center of the structure where they form the out-of-plane vortex core. ${ }^{31,32}$ The schematics of temporal XMCD and phase difference images of a Landau-flux closure state showing displacement of the vortex core upon the application of an alternating magnetic field are presented in Fig. 1(b). The temporal XMCD images $I_{0^{\circ}}$ and $I_{180^{\circ}}$ show the domain configuration at two phases of the excitation $\left(0^{\circ}\right.$ and $180^{\circ}$, respectively) while the phase difference image $\left(I_{0^{\circ}}-I_{180^{\circ}}\right)$ emphasizes the time-dependent effects, showing the extent of in-plane domain wall dynamics.

\section{Micromagnetic simulations}

Micromagnetic simulations were carried out using OOMMF, ${ }^{33}$ which solves the Landau-Lifshitz-Gilbert equation to calculate the temporal evolution of magnetic moments. At equilibrium, our experimental magnetic squares show an elongated domain wall along the $x$ axis at the vortex core due to a uniaxial anisotropy, which we believe is likely to be due to strain in the $\mathrm{Si}_{3} \mathrm{~N}_{4}$ membrane. We performed simulations of the equilibrium state for squares with side length $\ell=0.5 \mu \mathrm{m}$, kept small to reduce the simulation time. The equilibrium state was obtained by allowing an initial random magnetic 
(a)

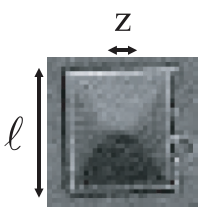

$4 \mu \mathrm{m}$

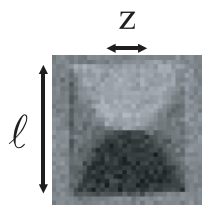

$6 \mu \mathrm{m}$

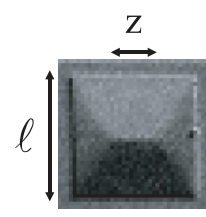

$8 \mu \mathrm{m}$

(b)

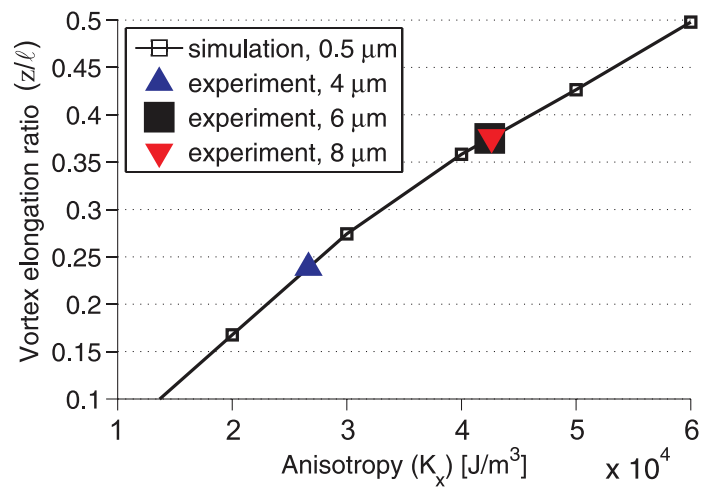

(c)

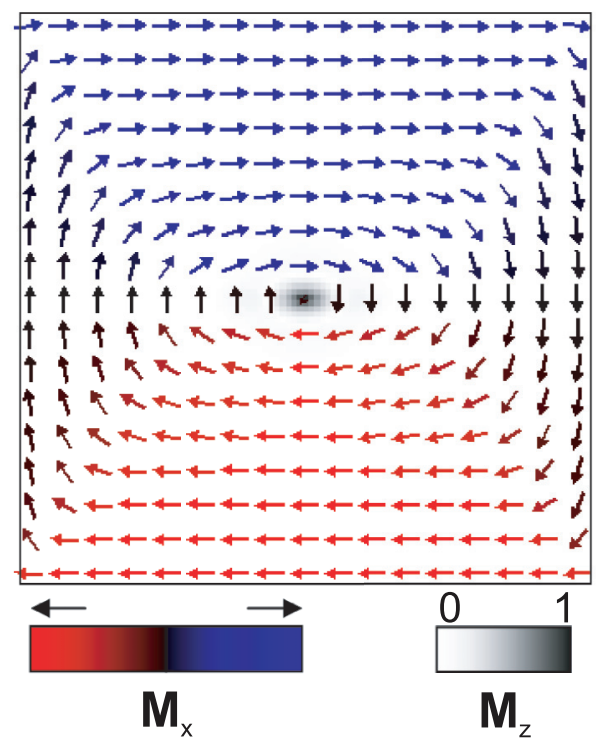

FIG. 2. (Color online) (a) XMCD images of the ground-state magnetization indicating domain wall elongation at the vortex $(z)$ induced by a uniaxial anisotropy $K_{x}$ in squares of side length $\ell=4$, 6 , and $8 \mu \mathrm{m}$. (b) The vortex elongation ratio $z / \ell$ for simulations of the ground state in $0.5 \mu \mathrm{m}$ squares is plotted against uniaxial anisotropy constants $K_{x}$. Experimentally derived $z / \ell$ ratios are used to interpolate equivalent experimental $K_{x}$ values, marked onto the plot for each structure. (c) The simulated equilibrium state showing the components of magnetization. Vectors represent the magnetization components in the $x y$ plane, with contrast corresponding to the $\vec{M}_{x}$ component. The $\vec{M}_{z}$ component shown by the background contrast indicates the out-of-plane vortex core at the center of the square.

state to relax until $\left|\vec{M} \times \vec{H}_{\text {eff }}\right| / M_{S}^{2} \leqslant 1 \times 10^{-5}$, where $M_{S}$ is the saturation magnetization and the damping parameter $\alpha=1$. To determine the value of $K_{x}$ to be implemented for dynamic simulations, the equilibrium state was simulated for different $K_{x}$ and the ratio of the length of the domain wall at the vortex core to the square side length $(z / \ell)$ matched to the experiment [Fig. 2(b)]. Effective values for the simulation of the smaller structure size were also derived for resonance

frequency $f$ and amplitude $\vec{H}_{0}$ to maintain equivalence to the experiment. Dynamic simulations were carried out with a cell size of $2.5 \mathrm{~nm} \times 2.5 \mathrm{~nm} \times 40 \mathrm{~nm}$ and $\alpha=0.1$. We used $M_{S}=871 \times 10^{3} \mathrm{~A} / \mathrm{m}$, as measured by vibrating sample magnetometry on a reference film. Standard values for permalloy for the exchange constant $A=1.3 \times 10^{-11} \mathrm{~J} / \mathrm{m}^{3}$ and Landau-Lifshitz gyromagnetic ratio $\gamma=2.21 \times 10^{5} \mathrm{~m} / \mathrm{As}$ were used. ${ }^{33}$ Full dynamic simulations of the temporal evolution of the magnetism were then compared with the experimental results.

\section{RESULTS}

\section{A. Equilibrium configurations}

To determine the equilibrium magnetic configurations and to measure the extent of domain wall elongation at the vortex in our experimental squares, we acquired static XMCD images prior to our dynamic studies. The equilibrium configurations for 4, 6, and $8 \mu \mathrm{m}$ squares are presented in Fig. 2(a), showing magnetic configurations similar to the Landau flux-closure patterns expected in rectangular structures. ${ }^{34,35}$ From our simulations an approximately linear relationship between $K_{x}$ versus $z / \ell$ was found, as shown in Fig. 2(b). From this relationship we could then determine an appropriate $K_{x}$ value to use in dynamic simulations. In our simulations using a side length $\ell=0.5 \mu \mathrm{m}$, interpolated values of $K_{x}$ for experimental 4,6 , and $8 \mu \mathrm{m}$ squares correspond to the uniaxial anisotropy constants of $K_{x}=26.7 \mathrm{~kJ} / \mathrm{m}^{3}, 42.4 \mathrm{~kJ} / \mathrm{m}^{3}$, and $42.6 \mathrm{~kJ} / \mathrm{m}^{3}$, respectively. A value of $K_{x}=42.0 \mathrm{~kJ} / \mathrm{m}^{3}$ has been used in the dynamic simulations for a comparison with the dynamics observed in the larger experimental structures. The simulated results will be discussed following an initial presentation of the experimental results.

Before proceeding, we show the magnetic configuration resulting from our simulation in Fig. 2(c). The components of magnetization at equilibrium for the simulated square are given. The vectors represent the magnetization components in the $x y$ plane, with the contrast corresponding to the $\vec{M}_{x}$ component. These vectors indicate a Néel wall at the center of the square resulting from the induced uniaxial anisotropy. The $\vec{M}_{z}$ component is shown by the background contrast of Fig. 2(c) and indicates a single vortex core with positive polarity, which sits in the middle of the domain wall at the center of the square.

\section{B. Dynamic stabilization of nonequilibrium configurations}

First, a low amplitude CW excitation was applied to our experimental structures to induce dynamics in the equilibrium state in the linear regime. Experimental phase difference images $\left(I_{0^{\circ}}-I_{180^{\circ}}\right)$ of the dynamics show the change in $\vec{M}_{x}(\vec{r})$ for our three squares $(4,6,8 \mu \mathrm{m})$ as $\vec{H}_{0}$ is increased (Fig. 3). The bright (dark) regions correspond to an increase (decrease) of the $\vec{M}_{x}$ component during excitation. At low $\vec{H}_{0}(\leqslant 1.60, \leqslant 1.75$, and $\leqslant 0.88 \mathrm{mT}$, respectively, for 4,6 , and $8 \mu \mathrm{m}$ squares), a cross similar to that associated with the dynamics of a Landau state as schematically shown in Fig. 1(b) is observed for all our structures [Fig. 3 (row A)]. At moderate $\vec{H}_{0}$ (especially at $3.00,2.65$, and $1.25 \mathrm{mT}$ ) a regime 


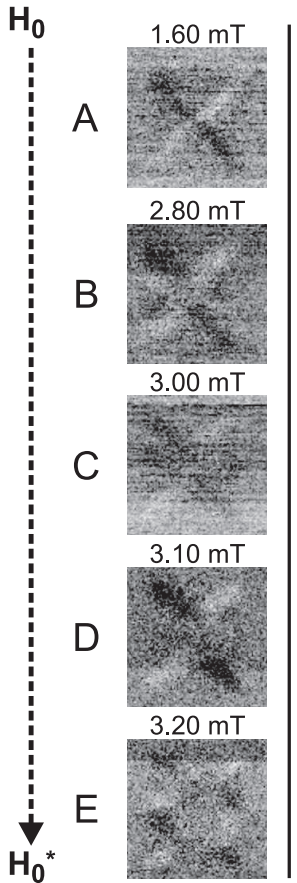

(a) $4 \mu \mathrm{m}$

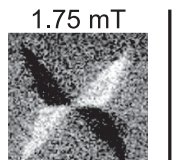

$2.20 \mathrm{mT}$

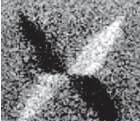

$2.65 \mathrm{mT}$
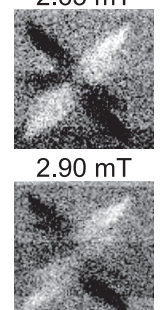

$3.10 \mathrm{mT}$

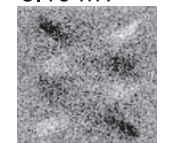

(b) $6 \mu \mathrm{m}$

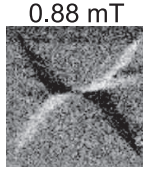

$1.25 \mathrm{mT}$
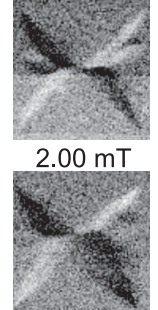

$2.50 \mathrm{mT}$

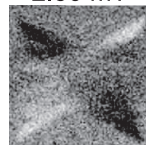

$2.80 \mathrm{mT}$

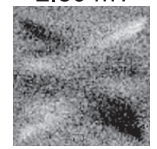

(c) $8 \mu \mathrm{m}$
FIG. 3. Phase difference magnetic images of (a) 4, (b) 6 , and (c) $8 \mu \mathrm{m}$ squares versus increasing excitation field amplitude. The transition from a simple cross shape to a more complex double cross shape at $\vec{H}_{0}^{*}$ indicates a transition to a nonequilibrium domain configuration.

of nonequilibrium states is entered where additional regions of the domain wall dynamics become apparent [Fig. 3 (rows $\mathrm{B}$ and C)]. Interestingly, we note that images similar to those of rows B and C in Fig. 3 were published in the work by Puzic et al.: ${ }^{18}$ phase difference images of a $2 \mu \mathrm{m} \times 2 \mu \mathrm{m}$ Permalloy square with a Landau pattern (without any apparent uniaxial anisotropy) show smaller regions of the domain wall dynamics in addition to the standard expected dynamic pattern. With our $\mathrm{CW}$ excitation, we have stabilized these nonequilibrium states and demonstrate greater lateral displacement of domain walls at higher fields (3.10, 2.90, and $2.50 \mathrm{mT}$ ) [Fig. 3 (row D)]. These nonequilibrium dynamic states are a precursor to states induced at a threshold field amplitude $\vec{H}_{0}^{*}(3.20,3.10$, and $2.80 \mathrm{mT}$ ), where we observe in all three structures a more complex dynamic response, with the signature of domain wall dynamics appearing as two cross-like regions of contrast [Fig. 3 (row E)]. The phase difference images at $\vec{H}_{0}^{*}$ are representative of a new magnetic configuration consisting of an induced nonequilibrium multidomain state with at least twice as many domains than the four domains found at equilibrium.

On comparing the magnitude of the threshold amplitudes required to enter nonequilibrium configurations for the three squares presented in Fig. 3 (row E), it is evident that larger structure sizes require smaller threshold amplitudes to reach the same configurations. When the excitation is removed, in all cases the squares return to the initial equilibrium configuration as in Fig. 2(a). The sense of gyration (clockwise/anticlockwise) of the excited vortex core is given by the core polarity $( \pm 1)$ and associated with a specific contrast in the phase difference images (black/white). If the core polarity were to change, the sense of gyration would also change and the contrast would be reversed. No such changes were observed during the experiments, thus the polarity of the vortex core in the equilibrium state was preserved.

\section{Comparison with simulation}

To understand the changes in the magnetic configuration under $\mathrm{CW}$ excitation we acquire temporal XMCD images. They allow us to directly observe the low amplitude response, the nonequilibrium topology at $\vec{H}_{0}^{*}$, and, importantly, the change in magnetic configuration at fields leading up to nonequilibrium configurations. The full set of temporal XMCD and phase difference images from 6- and 8- $\mu \mathrm{m}$ structures are displayed in Figs. 4(a) and 4(b). Note that these images were acquired on the same sample during a separate experiment to those of Fig. 3, hence the slightly different field values. For comparison with the experimental dynamic behavior, images with equivalent configurations from micromagnetic simulations are presented in Fig. 4(c). For these simulations, the derived resonance frequency $f=540 \mathrm{MHz}$ was used with $\vec{H}_{0}$ varied between 12.5-175.9 mT. Higher field and frequency values are required in the simulation for consistency with the experiment due to the smaller lateral size. Additionally, the simulation does not take into account the effect of magnetization reduction due to heating that could reduce the field amplitude required for a particular dynamic response. Resistive temperature measurements in similar experiments indicated that field strengths up to $0.9 \mathrm{mT}$ correspond to a sample temperature of $164^{\circ} \mathrm{C}$. Note also that, while we have incorporated a uniaxial anisotropy $K_{x}$ into the simulation for a direct comparison with the experiment, similar dynamic behavior to that outlined in this section, culminating in a stable nonequilibrium domain configuration at $\vec{H}_{0}^{*}$, can be observed with $K_{x}=0$.

In all cases, at low $\vec{H}_{0}$ [Figs. 4(a), 4(b), and 4(c), row A] we resolve four large domains of different contrast: one white and one black $\left[\vec{M}_{x} \perp \vec{H}(t)\right]$ and two gray $\left[\vec{M}_{x} \| \vec{H}(t)\right]$. As $\vec{H}(t)$ increases $\left(0^{\circ} \rightarrow 90^{\circ}\right)$, one gray domain grows at the expense of the other. This translational oscillation is the linear response of the equilibrium dynamic state. In the experimental images our first observation of the magnetization response beyond the linear regime is domain nucleation appearing as whiskers of magnetic contrast, which extend from the meeting points of domain walls toward the edges of the square. This can be seen at $\vec{H}_{0}=2.6 \mathrm{mT}$ for the $6-\mu \mathrm{m}$ square and at $\vec{H}_{0}=1.0 \mathrm{mT}$ for the $8-\mu \mathrm{m}$ square [Figs. 4(a) row $\mathrm{C}$ and 4 (b) row B]. Similar features previously interpreted as standing wave excitations within domains ${ }^{21}$ may also be understood as excitation of the magnetization beyond the linear response observed at low $\vec{H}_{0}$, and are a precursor to the multidomain configurations of higher-order excitation modes, which we have stabilized and imaged.

As the excitation amplitude is increased further, we observe that the initial length of the elongated domain wall at the vortex core is reduced. This can be seen when comparing the XMCD images at $\vec{H}_{0}=2.6 \mathrm{mT}$ and $\vec{H}_{0}=1.9 \mathrm{mT}$, respectively, for the 6 and $8 \mu \mathrm{m}$ squares with the XMCD images at lower $\vec{H}_{0}$ [Figs. 4(a) and 4 (b) rows A to C]. The reduction of the elongated domain wall at the vortex core is a result of the 


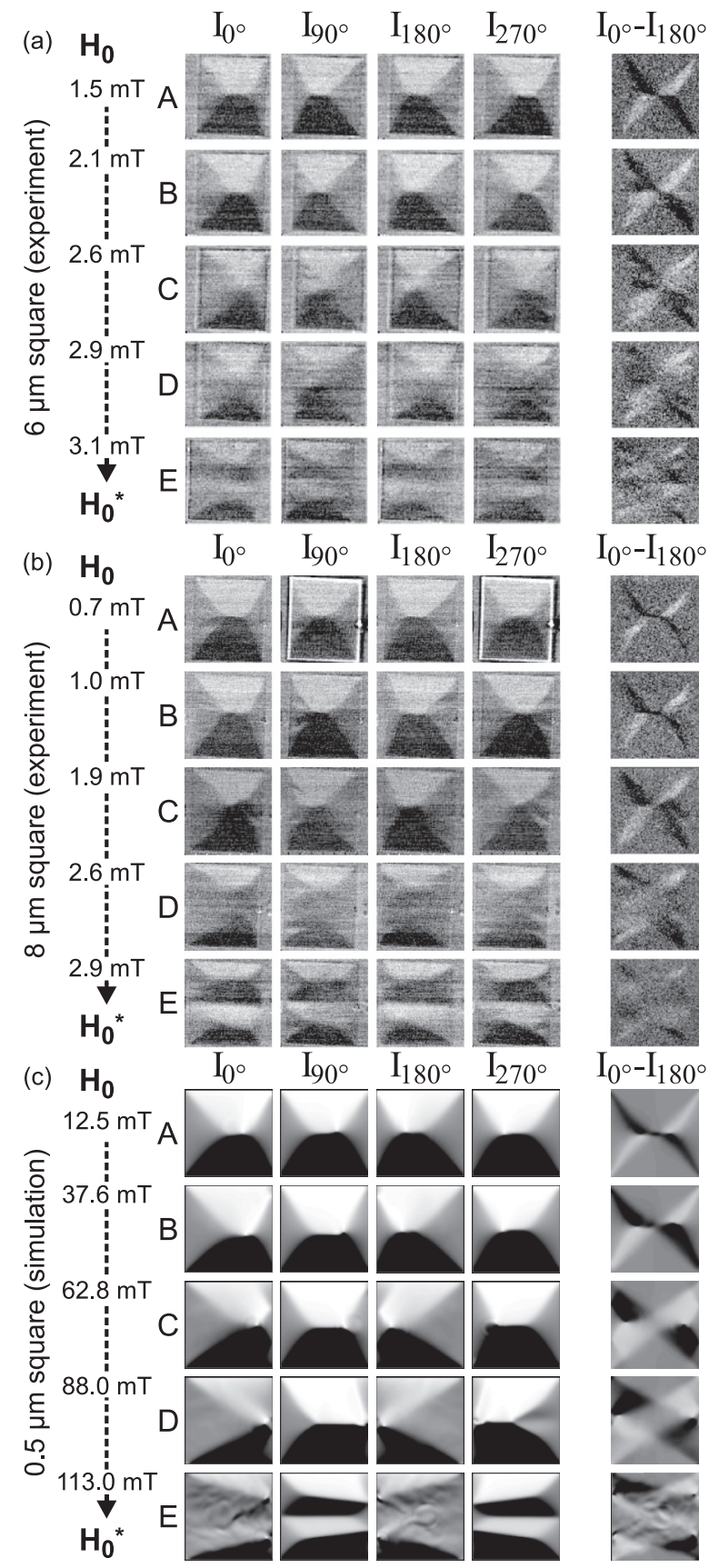

FIG. 4. Temporal XMCD images of the magnetization (at $0^{\circ}, 90^{\circ}$, $180^{\circ}$, and $270^{\circ}$ of excitation phase) and dynamic phase difference images $\left(I_{0^{\circ}}-I_{180^{\circ}}\right)$ for increasing excitation field amplitude $\vec{H}_{0}$ on structures of (a) $6 \mu \mathrm{m}$, (b) $8 \mu \mathrm{m}$, and (c) equivalent micromagnetic simulations for a $0.5-\mu \mathrm{m}$ structure.

growth of the gray domains $[\vec{M}$ parallel to $\vec{H}(t)]$ due to the applied magnetic field. This preferential modification of the gray domains and subsequent reduction in length of the central elongated domain wall is a result of a reduction of the Zeeman energy as $\vec{H}_{0}$ is increased. This behavior is confirmed in micromagnetic simulations.

Prior to the nonequilibrium configuration at $\vec{H}_{0}^{*}$, the experimental XMCD images show a domain configuration with washed out domains and poorly defined domain walls [Figs. 4(a) and 4(b) both row D]. In the temporal XMCD

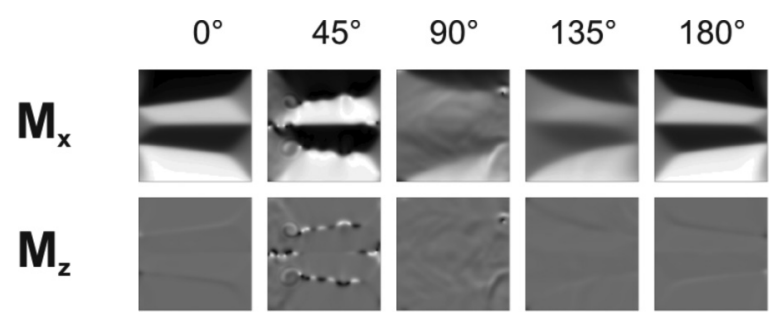

FIG. 5. Simulated $\vec{M}_{x}$ and $\vec{M}_{z}$ components of the magnetization every $45^{\circ}$ of phase for a half-period of excitation cycle at $\vec{H}_{0}^{*}=$ $113 \mathrm{mT}$.

images the large white and black domains, which are well resolved at lower amplitudes, appear to have decreased in size and at the center of the structure the magnetization configuration is not clear. Presenting the phase difference image $\left(I_{0^{\circ}}-I_{180^{\circ}}\right)$ alongside the XMCD images aids our interpretation of these unresolvable features: We are able to clarify that the whiskers of magnetization, which originate at points where domain walls meet, grow toward the edges of the square and act as a precursor to the separation of the white and black domains. These domains are observed to shrink from the center toward the edges of the square. This means that the area of domains with $\vec{M}$ parallel to $\vec{H}(t)$ is reduced.

At threshold fields, we observe clear nonequilibrium dynamic configurations in the XMCD images, showing the domain configurations of stable higher-order magnetic modes. For the 6- $\mu \mathrm{m}$ square, the nonequilibrium domain configuration and its distinct phase difference image is first observed at $\vec{H}_{0}^{*}=3.1 \mathrm{mT}$ [Fig. 4(a) row E]. It can be seen in the XMCD images that the number of domains has doubled along $\ell_{y}$. For the $8-\mu \mathrm{m}$ square, the first instance of the nonequilibrium configuration is observed at $2.9 \mathrm{mT}$ [Fig. 4(b) row E].

The simulations on the $0.5-\mu \mathrm{m}$ square indicate good qualitative agreement with the experimental data. Low amplitude dynamics correlate well to the experimental observations [Figs. 4(a), 4(b), and 4(c) row A]. The major feature of a higher-order nonequilibrium configuration at $\vec{H}_{0}^{*}$ is also evident in both the experiment and simulation [Figs. 4(a), 4(b), and 4(c) row E]. At intermediate fields of the simulation [Fig. 4(c) rows B to D] we observe the precursor to the nonequilibrium domain configuration, where domains grow toward the edges of the square with increasing field amplitude, ultimately causing the elongated domain wall at the vortex to collapse toward a single point at the edge.

Short period $(\lambda \sim \ell / 20)$ magnetic fluctuations observed in temporal XMCD images $\left(I_{0^{\circ}}\right.$ and $\left.I_{180^{\circ}}\right)$, and transferred to the phase difference image $\left(I_{0^{\circ}}-I_{180^{\circ}}\right)$ [Fig. 4(c) row E], are interpreted as spin wave emissions. Essentially, the energy pumped into the system with $\vec{H}_{0}^{*}$ causes a large amplitude motion of the domain walls and their motion is damped by the emission of spin waves. Such spin wave instabilities are known to appear as the excitation amplitude is increased beyond the linear precession threshold. ${ }^{28,34}$ The spin wave emissions are a recurring observation at each cycle of excitation and further details of this process are presented in Fig. 5. Here the contrast represents the $\vec{M}_{x}$ and $\vec{M}_{z}$ components of the magnetization at $45^{\circ}$ intervals for a half-period of excitation cycle at $\vec{H}_{0}^{*}=$ 113 mT. Looking at the $\vec{M}_{z}$ component we observe a 
(a)
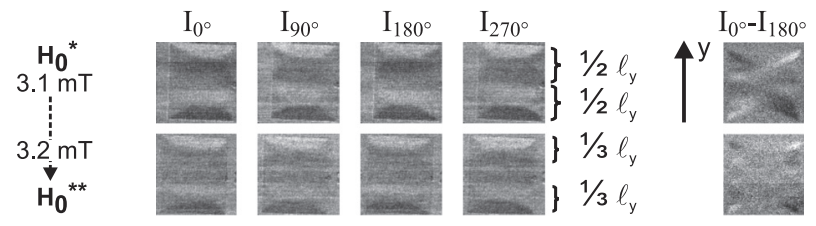

(b)
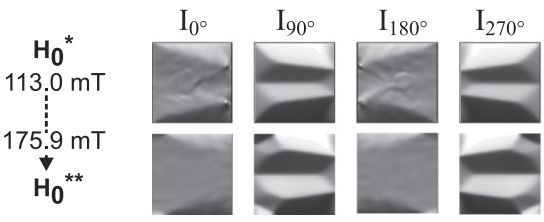

FIG. 6. Temporal XMCD images of the magnetization $\vec{M}_{x}(\vec{r})$ at field amplitudes above threshold values $\vec{H}_{0}^{*}$ for (a) experimental $8-\mu \mathrm{m}$ square and (b) micromagnetic simulation (equivalent parameters, $0.5-\mu \mathrm{m}$ square). The lateral extent of pairs of domains with respect to $\ell_{y}$ is marked on experimental images.

complex vortex substructure with multiple vortex creation and annihilation (see Fig. 5, 45 ${ }^{\circ}$ ) and the corresponding emission of spin waves visible in both $\vec{M}_{x}$ and $\vec{M}_{z}\left(45^{\circ}\right.$ and $\left.90^{\circ}\right)$.

\section{Excitation of higher-order modes}

We have observed evidence of even higher excitation modes both experimentally and in our simulated data. A change in the phase difference and temporal XMCD images recorded for the $8-\mu \mathrm{m}$ square is observed above $\vec{H}_{0}^{*}$, between $\vec{H}_{0}=$ $3.1 \mathrm{mT}$ and a higher threshold field $\vec{H}_{0}^{* *}=3.2 \mathrm{mT}$ (Ref. 36) [Fig. 6(a)]. In the phase difference image $\left(I_{0^{\circ}}-I_{180^{\circ}}\right)$ at $\vec{H}_{0}^{* *}$, the position and size of the black and white regions of contrast has changed with respect to that at $\vec{H}_{0}=3.1 \mathrm{mT}$. At $\vec{H}_{0}^{* *}$ we no longer observe the double cross-like dynamic signature in the phase difference image [Fig. 6(a)]. Instead, at $\vec{H}_{0}^{* *}=$ $3.2 \mathrm{mT}$ there is an increased number of smaller regions of magnetic contrast. In the temporal XMCD images, this can be seen as a reduction in size of the lower and upper pairs of domains. At $\vec{H}_{0}=3.1 \mathrm{mT}$ the lateral extent of these domains is half the vertical side length, i.e., $1 / 2 \ell_{y}$, reduced to $1 / 3 \ell_{y}$ at $\vec{H}_{0}^{* *}$, indicating the existence of a nonequilibrium magnetic configuration beyond that observed at $\vec{H}_{0}^{*}$.

Simulated data of the magnetization with $\vec{H}_{0}>\vec{H}_{0}^{*}$ is presented in Fig. 6(b). The phase difference images in the experiment and simulation indicate comparable dynamic behavior, with a similar change in the dynamic signature above threshold fields. As observed in the experiment [Fig. 6(a)], the phase difference image in the simulation shows that the double cross-like dynamic signature at $\vec{H}_{0}^{*}$ is replaced at $\vec{H}_{0}^{* *}$ by regions of dynamic contrast at the corners of the square and less clarity at the center of the structure. While the simulated temporal XMCD images do not match the experiment perfectly, we do observe a greater number of domains in the magnetic configuration at $\vec{H}_{0}^{* *}$. There is reduced clarity of spin wave contributions in the temporal XMCD images at higher $\vec{H}_{0}\left(\vec{H}_{0}^{* *}=175.9 \mathrm{mT}\right)$ and further nucleation of magnetic domains originating at the corners of the square.

\section{SUMMARY}

We have imaged Landau states with an elongated domain wall at the vortex core under $\mathrm{CW}$ excitation applied at a single frequency and observed what happened on increasing the field amplitude. At low amplitudes we observed the dynamics of the equilibrium Landau state. When increasing the amplitude to threshold values, a more complex nonequilibrium dynamic configuration was induced, with at least twice the number of domains than was observed at equilibrium. Such an emergence of higher-order modes observed in the spatial domain have been previously observed in the frequency domain, where they are referred to as the Suhl instabilities. ${ }^{14}$ Furthermore, when we reduced the amplitude from the threshold values, the equilibrium state was restored. The experimentally observed behavior is verified qualitatively via simulations, which provide additional insight into the mechanism of nonlinear energy losses that is observed to occur by spin wave emissions.

Previous studies have largely concentrated on frequency domain imaging and the spatial imaging of precessional modes using simulated and measured ferromagnetic resonance and optical techniques. ${ }^{5-7,9,11,12}$ These studies present the excitation of different spin wave modes, but not the change in the domain configuration that we see on changing the field amplitude. Our excitation and imaging methods therefore provide a complementary experiment, allowing high resolution spatial imaging to resolve the details of changes in the magnetization topology as the excitation amplitude is increased. We have presented a dynamic stabilization of excitation modes and, moreover, imaged the transformation of the magnetization from the equilibrium mode to a nonequilibrium mode. Analysis shows that the first sign of this transformation is nucleation and the growth of small domains from the vortex core toward the edges of the magnetic squares. There is also a decrease in the length of the domain wall that coincides with the vortex as the magnetic configuration is modified to reduce the Zeeman energy contribution.

Our detailed observations not only provide spatially resolved insight into the modal spectrum of micrometer-scale magnetic squares, but could also prove useful for vortex core switching. In particular, a reliable and controllable switching of the vortex core requires a deeper understanding of the nonlinear processes and a detailed knowledge of the dependence upon the frequency and amplitude of induced spin modes. Spatially resolved details of these processes, such as those outlined in this work, are therefore essential for further developments in this area.

\section{ACKNOWLEDGMENTS}

We are grateful to Benjamin Watts and Mirco Holler for support during STXM measurements. For assistance with sample fabrication, we acknowledge Anja Weber, Vitaliy Guzenko, Eugen Deckardt, and Michael Horisberger. Magnetic characterization was carried out by Christopher Morrison (University of Manchester). This work was supported by funding from the Swiss National Science Foundation (2000021_125039) and the PolLux end station was financed by the German Minister für Bildung und Forschung (BMBF), Contract No. $05 \mathrm{KS} 4 \mathrm{WE} 1 / 6$. 
${ }^{1}$ S. Bohlens, B. Krüger, A. Drews, M. Bolte, G. Meier, and D. Pfannkuche, Appl. Phys. Lett. 93, 142508 (2008).

${ }^{2}$ B. Pigeau, G. de Loubens, O. Klein, A. Riegler, F. Lochner, G. Schmidt, L. W. Molenkamp, V. S. Tiberkevich, and A. N. Slavin, Appl. Phys. Lett. 96, 132506 (2010).

${ }^{3}$ K. Nakano, D. Chiba, N. Ohshima, S. Kasai, T. Sato, Y. Nakatani, K. Sekiguchi, K. Kobayashi, and T. Ono, Appl. Phys. Lett. 99, 262505 (2011).

${ }^{4}$ K. Y. Guslienko, B. A. Ivanov, V. Novosad, Y. Otani, H. Shima, and K. Fukamichi, J. Appl. Phys. 91, 8037 (2002).

${ }^{5}$ M. Buess, R. Höllinger, T. Haug, K. Perzlmaier, U. Krey, D. Pescia, M. R. Scheinfein, D. Weiss, and C. H. Back, Phys. Rev. Lett. 93, 077207 (2004).

${ }^{6}$ M. Yan, G. Leaf, H. Kaper, R. Camley, and M. Grimsditch, Phys. Rev. B 73, 014425 (2006).

${ }^{7}$ M. Bailleul, R. Höllinger, K. Perzlmaier, and C. Fermon, Phys. Rev. B 76, 224401 (2007).

${ }^{8}$ K. Y. Guslienko, R. H. Heredero, and O. Chubykalo-Fesenko, Phys. Rev. B 82, 014402 (2010).

${ }^{9}$ I. Neudecker, K. Perzlmaier, F. Hoffmann, G. Woltersdorf, M. Buess, D. Weiss, and C. H. Back, Phys. Rev. B 73, 134426 (2006).

${ }^{10}$ A. Drews, B. Krüger, G. Selke, T. Kamionka, A. Vogel, M. Martens, U. Merkt, D. Möller, and G. Meier, Phys. Rev. B 85, 144417 (2012).

${ }^{11}$ R. D. McMichael and M. D. Stiles, J. Appl. Phys. 97, 10J901 (2005).

${ }^{12}$ V. E. Demidov, U.-H. Hansen, and S. O. Demokritov, Phys. Rev. Lett. 98, 157203 (2007).

${ }^{13}$ X. M. Cheng, K. S. Buchanan, R. Divan, K. Y. Guslienko, and D. J. Keavney, Phys. Rev. B 79, 172411 (2009).

${ }^{14}$ H. Suhl, J. Phys. Chem. Solids 1, 209 (1957).

${ }^{15}$ T. Gerrits, M. L. Schneider, A. B. Kos, and T. J. Silva, Phys. Rev. B 73, 094454 (2006).

${ }^{16}$ J. Podbielski, D. Heitmann, and D. Grundler, Phys. Rev. Lett. 99, 207202 (2007).

${ }^{17}$ H. Stoll, A. Puzic, B. van Waeyenberge, P. Fischer, J. Raabe, M. Buess, T. Haug, R. Hollinger, C. Back, D. Weiss, and G. Denbeaux, Appl. Phys. Lett. 84, 3328 (2004).

${ }^{18}$ A. Puzic, B. Van Waeyenberge, K. W. Chou, P. Fischer, H. Stoll, G. Schütz, T. Tyliszczak, K. Rott, H. Brückl, G. Reiss, I. Neudecker, T. Haug, M. Buess, and C. H. Back, J. Appl. Phys. 97, 10E704 (2005).

${ }^{19}$ J. Raabe, C. Quitmann, C. H. Back, F. Nolting, S. Johnson, and C. Buehler, Phys. Rev. Lett. 94, 217204 (2005).

${ }^{20}$ J. Stöhr and H. C. Siegmann, Magnetism: From Fundamentals to Nanoscale Dynamics, Springer Series in Solid-state Sciences S (Springer, New York, 2006).
${ }^{21}$ M. Buess, J. Raabe, C. Quitmann, J. Stahl, and C. H. Back, Surf. Sci. 601, 5246 (2007).

${ }^{22}$ B. Van Waeyenberge, A. Puzic, H. Stoll, K. W. Chou, T. Tyliszczak, R. Hertel, M. Fähnle, H. Brückl, K. Rott, G. Reiss, I. Neudecker, D. Weiss, C. H. Back, and G. Schütz, Nature (London) 444, 461 (2006).

${ }^{23}$ A. Vansteenkiste, K. W. Chou, M. Weigand, M. Curcic, V. Sackmann, H. Stoll, T. Tyliszczak, G. Woltersdorf, C. H. Back, G. Schütz, and B. Van Waeyenberge, Nat. Phys. 5, 332 (2009).

${ }^{24}$ A. Bisig, J. Rhensius, M. Kammerer, M. Curcic, H. Stoll, G. Schutz, B. Van Waeyenberge, K. W. Chou, T. Tyliszczak, L. J. Heyderman, S. Krzyk, A. von Bieren, and M. Klaui, Appl. Phys. Lett. 96, 152506 (2010).

${ }^{25}$ A. Vogel, T. Kamionka, M. Martens, A. Drews, K. W. Chou, T. Tyliszczak, H. Stoll, B. Van Waeyenberge, and G. Meier, Phys. Rev. Lett. 106, 137201 (2011).

${ }^{26}$ J. Raabe, G. Tzvetkov, U. Flechsig, M. Böge, A. Jaggi, B. Sarafimov, M. G. C. Vernooij, T. Huthwelker, H. Ade, D. Kilcoyne, T. Tyliszczak, R. H. Fink, and C. Quitmann, Rev. Sci. Instrum. 79, 113704 (2008).

${ }^{27}$ K. Jefimovs, J. Vila-Comamala, T. Pilvi, J. Raabe, M. Ritala, and C. David, Phys. Rev. Lett. 99, 264801 (2007).

${ }^{28}$ A. Puzic, T. Korhonen, B. Kalantari, J. Raabe, C. Quitmann, P. Jüllig, L. Bommer, D. Goll, G. Schütz, S. Wintz, T. Strache, M. Körner, D. Markó, C. Bunce, and J. Fassbender, Synchrotron Radiation News 23, 26 (2010).

${ }^{29}$ G. Schütz, W. Wagner, W. Wilhelm, P. Kienle, R. Zeller, R. Frahm, and G. Materlik, Phys. Rev. Lett. 58, 737 (1987).

${ }^{30}$ A. Rogalev, F. Wilhelm, N. Jaouen, J. Goulon, and J.-P. Kappler, in Magnetism: A Synchrotron Radiation Approach, Lecture Notes in Physics, Vol. 697 (Springer, Berlin, 2006), pp. 71-93.

${ }^{31}$ A. Wachowiak, J. Wiebe, M. Bode, O. Pietzsch, M. Morgenstern, and R. Wiesendanger, Science 298, 577 (2002).

${ }^{32}$ K. Kuepper, S. Wintz, J. Raabe, M. Buess, C. Akhmadaliev, L. Bischoff, C. Quitmann, and J. Fassbender, J. Phys.: Condens. Matter 21, 436003 (2009).

${ }^{33}$ M. J. Donahue and D. G. Porter, oommF User's Guide, Version 1.0, Interagency Report NISTIR 6376 (National Institute of Standards and Technology, Gaithersburg, MD, 1999).

${ }^{34}$ S. Hankemeier, R. Frömter, N. Mikuszeit, D. Stickler, H. Stillrich, S. Pütter, E. Y. Vedmedenko, and H. P. Oepen, Phys. Rev. Lett. 103, 147204 (2009).

${ }^{35}$ R. Hertel, O. Fruchart, S. Cherifi, P. O. Jubert, S. Heun, A. Locatelli, and J. Kirschner, Phys. Rev. B 72, 214409 (2005).

${ }^{36} \mathrm{Phase}$ difference images were acquired during an earlier experiment than the temporal XMCD images, but on the same sample with the same excitation and imaging parameters (within 10\%). 